\title{
Multiobjective Optimal VAR Dispatch Using Strength Pareto Evolutionary Algorithm
}

\author{
M. A. Abido, Member, IEEE
}

\begin{abstract}
In this paper, Strength Pareto Evolutionary Algorithm (SPEA) for optimal reactive power (VAR) dispatch problem is presented. The optimal VAR dispatch problem is formulated as a nonlinear constrained multiobjective optimization problem where the real power loss and the voltage stability are to be optimized simultaneously. The proposed approach handles the problem as a true multiobjective optimization problem. A hierarchical clustering algorithm is imposed to provide the decision maker with a representative and manageable Pareto-optimal set. Moreover, fuzzy set theory is employed to extract the best compromise solution over the tradeoff curve. The results demonstrate the capabilities of the proposed approach to generate true and well-distributed Paretooptimal solutions of the multiobjective VAR dispatch problem in one single run. In addition, the effectiveness of the proposed approach and its potential to solve the multiobjective VAR dispatch problem are confirmed.
\end{abstract}

Index Terms - Optimal VAR dispatch, Evolutionary algorithms, Multiobjective optimization

\section{INTRODUCTION}

$\mathrm{I}^{\mathrm{N}}$ $\mathrm{N}$ the past two decades, the problem of reactive power control for improving economy and security of power system operation has received much attention. Generally, the voltage stability can be enhanced by reallocating reactive power generations in the system. This can be achieved by adjusting transformer taps, generator voltages, and switchable VAR sources. In addition, the system losses can be minimized via redistribution of reactive power in the system. Therefore, the problem of the reactive power dispatch can be optimized to enhance the voltage stability and minimize the system losses as well.

Several methods to solve the optimal reactive power dispatch problem have been proposed in the literature. Generally, there are three approaches to solve this complex problem. The first approach employs nonlinear programming technique [1]. However, nonlinear programming based procedures have many drawbacks, such as insecure convergence properties, long execution time, and algorithmic complexity. The second approach uses sensitivity analysis and gradient-based optimization algorithms by linearizing the objective function and the system constraints around an operating point [2]. Obviously, this problem is highly complex

M. A. Abido is with Electrical Engineering Department, King Fahd University of Petroleum and Minerals (e-mail: mabido@kfupm.edu.sa). optimization problem and may have several local minima. In this case, the gradient-based methods are susceptible to be trapped in local minima and the solution obtained will not be the optimal one. Moreover, calculation of the sensitivity factors is a time consuming process and inefficient for largescale system applications. The third approach utilizes the heuristic methods to search for the optimal solution in the problem space [3-6]. It has been theoretically proved that these methods converge to the optimal solution with probability one provided that certain conditions are satisfied. These heuristic methods have been applied to solve the optimal VAR dispatch problem with impressive success.

Recently, the VAR dispatch problem is formulated as a multiobjective optimization problem. However, the problem is not treated as a true multiobjective problem [7-8]. It was converted to a single objective problem by linear combination of different objectives as a weighted sum [7]. Unfortunately, this requires multiple runs as many times as the number of desired Pareto-optimal solutions. Furthermore, this method cannot be used to find Pareto-optimal solutions in problems having a non-convex Pareto-optimal front. In addition, there is no rational basis of determining adequate weights and the objective function so formed may lose significance due to combining non-commensurable objectives. To avoid this difficulty, the $\varepsilon$-constraint method for multiobjective optimization was presented in [8]. This method is based on optimization of the most preferred objective and considering the other objectives as constraints bounded by some allowable levels $\varepsilon$. These levels are then altered to generate the entire Pareto-optimal set. The most obvious weaknesses of this approach are that it is time-consuming and tends to find weakly nondominated solutions.

On the contrary, the studies on evolutionary algorithms, over the past few years, have shown that these methods can be efficiently used to eliminate most of the difficulties of classical methods [9-11]. Since they use a population of solutions in their search, multiple Pareto-optimal solutions can, in principle, be found in one single run. Recently, some preliminary successful results of optimal VAR dispatch have been reported [12] where minimizing voltage differences has been considered as an objective. This could be detrimental since it utilizes scarce reactive resources for no stability or operational benefit.

In this paper, the Strength Pareto Evolutionary Algorithm (SPEA) based approach is proposed for solving the 
multiobjective VAR dispatch optimization problem. The problem is formulated as a nonlinear constrained multiobjective optimization problem where the real power loss and voltage stability objectives are optimized simultaneously. A hierarchical clustering technique is implemented to provide the power system operator with a representative and manageable Pareto-optimal set. Moreover, a fuzzy-based mechanism is employed to extract the best compromise solution over the trade-off curve. The effectiveness and potential of the proposed approach to solve the multiobjective VAR dispatch problem are demonstrated.

\section{Problem Formulation}

The optimal VAR dispatch problem is to optimize the steady state performance of a power system in terms of one or more objective functions while satisfying several equality and inequality constraints. Generally the problem can be formulated as follows.

\section{A. Objective Functions}

Real Power Loss $\left(P_{\underline{L}}\right)$ : This objective is to minimize the real power loss in transmission lines that can be expressed as

$J_{1}=P_{L}=\sum_{k=1}^{n l} g_{k}\left[V_{i}^{2}+V_{j}^{2}-2 V_{i} V_{j} \cos \left(\delta_{i}-\delta_{j}\right)\right]$

where $n l$ is the number of transmission lines; $g_{k}$ is the conductance of the $k^{\text {th }}$ line; $V_{i} \angle \delta_{i}$ and $V_{j} \angle \delta_{j}$ are the voltages at the end buses $i$ and $j$ of the $k^{\text {th }}$ line respectively.

Voltage Stability Index: In this study, voltage stability enhancement is achieved through minimizing the voltage stability indicator $L$-index [13-14] values at every bus of the system and consequently the global power system $L$-index. The indicator value varies in the range between 0 (the no load case) and 1 which corresponds to voltage collapse. For multinode system

$I_{\text {bus }}=Y_{\text {bus }} \times V_{\text {bus }}$

By segregating the load buses (PQ) from generator buses (PV), Eq. (2) can write as

$$
\begin{aligned}
& {\left[\begin{array}{l}
I_{L} \\
I_{G}
\end{array}\right]=\left[\begin{array}{ll}
Y_{1} & Y_{2} \\
Y_{3} & Y_{4}
\end{array}\right]\left[\begin{array}{l}
V_{L} \\
V_{G}
\end{array}\right]} \\
& {\left[\begin{array}{c}
V_{L} \\
I_{G}
\end{array}\right]=\left[\begin{array}{ll}
H_{1} & H_{2} \\
H_{3} & H_{4}
\end{array}\right]\left[\begin{array}{l}
I_{L} \\
V_{G}
\end{array}\right]}
\end{aligned}
$$

$V_{L}, I_{L}$ : Voltages and Currents for PQ buses

$V_{G}, I_{G}$ : Voltages and Currents for PV buses

Where, $\mathrm{H}_{1}, \mathrm{H}_{2}, \mathrm{H}_{3}, \mathrm{H}_{4}$ : submatrices generated from $Y_{\text {bus }}$ partial inversion. Let

$$
\bar{V}_{o k}=\sum_{i=1}^{n G} H_{2 k i} \cdot \bar{V}_{i}
$$

$n_{G}$ : number of generators

$$
\begin{aligned}
& H_{2}=-Y_{1} \times Y_{2} \\
& L_{k}=\left|1+\frac{V_{o k}}{V_{k}}\right|
\end{aligned}
$$

$L_{k}: L$-index voltage stability indicator for bus $k$. Stability requires that $L_{k}<1$ and must not be violated on a continuous basis. Hence a global system indicator describing the stability of the complete system is $L_{\max }=\max \left\{L_{k}\right\}$, where in $\left\{L_{k}\right\}$ all load bus indices are listed.

The objective is to minimize $L_{\max }$, that is, $J_{2}=\max \left\{L_{k} ; k=1,2, \ldots\right.$, number of buses $\}$

\section{B. Problem Constraints}

Equality Constraints: These constraints represent typical load flow equations as follows.

$$
P_{G_{i}}-P_{D_{i}}-V_{i} \sum_{j=1}^{N B} V_{j}\left[G_{i j} \cos \left(\delta_{i}-\delta_{j}\right)+B_{i j} \sin \left(\delta_{i}-\delta_{j}\right)\right]=0
$$

$$
Q_{G_{i}}-Q_{D_{i}}-V_{i} \sum_{j=1}^{N B} V_{j}\left[G_{i j} \sin \left(\delta_{i}-\delta_{j}\right)-B_{i j} \cos \left(\delta_{i}-\delta_{j}\right)\right]=0
$$

(10)

where $i=1, \ldots N B, N B$ is the number of buses; $P_{G}$ and $Q_{G}$ are the generator real and reactive power respectively; $P_{D}$ and $Q_{D}$ are the load real and reactive power respectively; $G_{i j}$ and $B_{i j}$ are the transfer conductance and susceptance between bus $i$ and bus $j$ respectively.

Inequality Constraints: These constraints represent the system operating constraints as follows.

Generation constraints: Generator voltages $V_{G}$ and reactive power outputs $Q_{G}$ are restricted by their lower and upper limits as follows:

$V_{G_{i}}^{\min } \leq V_{G_{i}} \leq V_{G_{i}}^{\max }, i=1, \ldots, N G$

$Q_{G_{i}}^{\min } \leq Q_{G_{i}} \leq Q_{G_{i}}^{\max }, i=1, \ldots, N G$

(12)

where $N G$ is number of generators.

Transformer constraints: Transformer tap $T$ settings are bounded as follows:

$T_{i}^{\min } \leq T_{i} \leq T_{i}^{\max }, i=1, \ldots, N T$

where $N T$ is the number of transformers.

Switchable VAR sources constraints: Switchable VAR compensations $Q_{C}$ are restricted by their limits as follows:

$Q_{c i}^{\min } \leq Q_{c i} \leq Q_{c i}^{\max }, i=1, \ldots, N C$

(14)

where $N C$ is the number of switchable VAR sources.

Load Bus Voltage: These include the constraints of voltages at load buses $V_{L}$ as follows:

$V_{L_{i}}^{\min } \leq V_{L_{i}} \leq V_{L_{i}}^{\max }, i=1, \ldots, N L$

\section{Formulation}

Aggregating the objectives and constraints, the problem can be mathematically formulated as a nonlinear constrained multiobjective optimization problem as follows.

Minimize $\left[J_{1}, J_{2}\right]$

Subject to:

$g(\mathrm{x}, \mathrm{u})=0$

$h(\mathrm{x}, \mathrm{u}) \leq 0$

where:

$\mathrm{x}$ : is the vector of dependent variables consisting of load bus voltages $V_{L}$, generator reactive power outputs $Q_{G}$, and transmission line loadings $S_{l}$. Hence, x can be expressed as 


$$
\mathrm{x}^{T}=\left[V_{L_{1}} \ldots V_{L_{N L}}, Q_{G_{1}} \ldots Q_{G_{N G}}\right]
$$

$\mathrm{u}$ : is the vector of control variables consisting of generator voltages $V_{G}$, transformer tap settings $T$, and shunt compensations $Q_{c}$. Hence, u can be expressed as

$$
\mathrm{u}^{T}=\left[V_{G_{1}} \ldots V_{G_{N G}}, T_{1} \ldots T_{N T}, Q_{c 1} \ldots Q_{c_{N C}}\right]
$$

$g:$ is the equality constraints.

$h$ : is the inequality constraints.

\section{Multiobjective Optimization}

Many real-world problems involve simultaneous optimization of several objective functions. Generally, these functions are non-commensurable and often competing objectives. Multiobjective optimization with such objective functions gives rise to a set of optimal solutions, instead of one optimal solution. The reason for the optimality of many solutions is that no one can be considered to be better than any other with respect to all objective functions. These optimal solutions are known as Pareto-optimal solutions.

A general multiobjective optimization problem consists of a number of objectives to be optimized simultaneously and is associated with a number of equality and inequality constraints. It can be formulated as follows:

$$
\begin{array}{ll}
\text { Minimize } & f_{i}(x) i=1, \ldots, N_{o b j} \\
\text { Subject to }:\left\{\begin{array}{ll}
g_{j}(x)=0 & j=1, \ldots, M \\
h_{k}(x) \leq 0 & k=1, \ldots, K
\end{array}\right. \text { Constraints }
\end{array}
$$

where $f_{i}$ is the $i^{\text {th }}$ objective functions, $x$ is a decision vector that represents a solution, and $N_{o b j}$ is the number of objectives.

For a multiobjective optimization problem, any two solutions $x^{1}$ and $x^{2}$ can have one of two possibilities- one covers or dominates the other or none dominates the other. In a minimization problem, without loss of generality, a solution $x^{1}$ dominates $x^{2}$ iff the following two conditions are satisfied:

1. $\forall i \in\left\{1,2, \ldots, N_{o b j}\right\}: f_{i}\left(x^{1}\right) \leq f_{i}\left(x^{2}\right)$

2. $\exists j \in\left\{1,2, \ldots, N_{o b j}\right\}: f_{j}\left(x^{1}\right)<f_{j}\left(x^{2}\right)$

If any of the above conditions is violated, the solution $x^{1}$ does not dominate the solution $x^{2}$. If $x^{1}$ dominates the solution $x^{2}, x^{1}$ is called the nondominated solution. The solutions that are nondominated within the entire search space are denoted as Pareto-optimal and constitute the Pareto-optimal set or Pareto-optimal front.

\section{THE PROPOSED APPROACH}

\section{A. Overview}

Recently, the studies on evolutionary algorithms have shown that these algorithms can be efficiently used to eliminate most of the difficulties of classical methods that can be summarized as:

- An algorithm has to be applied many times to find multiple Pareto-optimal solutions.

- Most algorithms demand some knowledge about the problem being solved.

- Some algorithms are sensitive to the shape of the Pareto-optimal front.

- The spread of Pareto-optimal solutions depends on efficiency of the single objective optimizer.

In general, the goal of a multiobjective optimization algorithm is not only guide the search towards the Pareto-optimal front but also maintain population diversity in the set of the nondominated solutions.

\section{B. Strength Pareto Evolutionary Algorithm (SPEA)}

The proposed SPEA based approach has the following features [11]: -

- It stores externally those individuals that represent a nondominated front among all solutions considered so far.

- It uses the concept of Pareto dominance in order to assign scalar fitness values to individuals.

- It performs clustering to reduce the number of individuals externally stored without destroying the characteristics of the trade-off front.

Generally, the algorithm can be described in the following steps.

Step 1 (Initialization): Generate an initial population and create an empty external Pareto-optimal set.

Step 2 (External set updating): The external Pareto-optimal set is updated as follows.

(a) Search the population for the nondominated individuals and copy them to the external Pareto set.

(b) Search the external Pareto set for the nondominated individuals and remove all dominated solutions from the set.

(c) If the number of the individuals externally stored in the Pareto set exceeds the prespecified maximum size, reduce the set by clustering.

Step 3 (Fitness assignment): Calculate the fitness values of individuals in both external Pareto set and the population as follows.

(a) Assign a real value $s \in[0,1)$ called strength for each individual in the Pareto optimal set. The strength of an individual is proportional to the number of individuals covered by it. The strength of a Pareto solution is at the same time its fitness.

(b) The fitness of each individual in the population is the sum of the strengths of all external Pareto solutions by which it is covered. In order to guarantee that Pareto solutions are most likely to be produced, a small positive number is added to the resulting value.

Step 4 (Selection): Combine the population and the external set individuals. Select two individuals at random and compare their fitness. Select the better one and copy it to the mating pool.

Step 5 (Crossover and Mutation): Perform the crossover and mutation operations according to their probabilities to generate the new population.

Step 7 (Termination): Check for stopping criteria. If any one is satisfied then stop else copy new population to old population and go to Step 2. In this study, the search will be stopped if the generation counter exceeds its maximum number.

\section{Reducing Pareto Set by Clustering}

In some problems, the Pareto optimal set can be extremely large. In this case, reducing the set of nondominated solutions without destroying the characteristics of the trade-off 
front is desirable from the decision maker's point of view. An average linkage based hierarchical clustering algorithm [15] is employed to reduce the Pareto set to manageable size. It works iteratively by joining the adjacent clusters until the required number of groups is obtained. It can be described as: given a set $P$ which its size exceeds the maximum allowable size $N$, it is required to form a subset $P^{*}$ with the size $N$. The algorithm is illustrated in the following steps.

Step 1: Initialize cluster set $C$; each individual $i \in P$ constitutes a distinct cluster.

Step 2: If number of clusters $\leq N$, then go to Step 5, else go to Step 3.

Step 3: Calculate the distance of all possible pairs of clusters. The distance $d_{c}$ of two clusters $c_{1}$ and $c_{2} \in C$ is given as the average distance between pairs of individuals across the two clusters

$$
d_{c}=\frac{1}{n_{1} \cdot n_{2}} \sum_{i_{1} \in c_{1}, i_{2} \in c_{2}} d\left(i_{1}, i_{2}\right)
$$

where $n_{1}$ and $n_{2}$ are the number of individuals in the clusters $c_{1}$ and $c_{2}$ respectively. The function $d$ reflects the distance in the objective space between individuals $i_{1}$ and $i_{2}$.

Step 4: Determine two clusters with minimal distance $d_{c}$. Combine them into a larger one. Go to Step 2.

Step 5: Find the centroid of each cluster. Select the nearest individual in this cluster to the centroid as a representative individual and remove all other individuals from the cluster.

Step 6: Compute the reduced nondominated set $P^{*}$ by uniting the representatives of the clusters.

\section{Best Compromise Solution}

Upon having the Pareto-optimal set of nondominated solution, the proposed approach presents one solution to the decision maker as the best compromise solution. Due to imprecise nature of the decision maker's judgment, the $i$-th objective function $F_{i}$ is represented by a membership function $\mu_{i}$ defined as [16]

$$
\mu_{i}= \begin{cases}1 & F_{i} \leq F_{i}^{\min } \\ \frac{F_{i}^{\max }-F_{i}}{F_{i}^{\max }-F_{i}^{\min }} & F_{i}^{\min }<F_{i}<F_{i}^{\max } \\ 0 & F_{i} \geq F_{i}^{\max }\end{cases}
$$

where $F_{i}^{\min }$ and $F_{i}^{\max }$ are the minimum and maximum value of the $i$-th objective function among all nondominated solutions, respectively.

For each nondominated solution $k$, the normalized membership function $\mu^{k}$ is calculated as

$$
\mu^{k}=\frac{\sum_{i=1}^{N_{o b j}} \mu_{i}^{k}}{\sum_{k=1}^{M} \sum_{i=1}^{N_{o b j}} \mu_{i}^{k}}
$$

where $M$ is the number of nondominated solutions. The best compromise solution is that having the maximum value of $\mu^{k}$.

\section{IMPLEMENTATION OF THE Proposed APPROACH}

\section{A. The Computational Flow}

In this study, the basic SPEA has been developed in order to make it suitable for solving real-world nonlinear constrained optimization problems. The following modifications have been incorporated in the basic algorithm.

(a) A procedure is imposed to check the feasibility of the initial population individuals and the generated children through GA operations. This ensures the feasibility of Pareto-optimal nondominated solutions.

(b) A procedure for updating the Pareto-optimal set is developed. In every generation, the nondominated solutions in the first front are combined with the existing Pareto-optimal set. The augmented set is processed to extract its nondominated solutions that represent the updated Pareto-optimal set.

(c) A fuzzy-based mechanism is employed to extract the best compromise solution over the trade-off curve and assist the decision maker to adjust the VAR sources efficiently.

The computational flow of the proposed SPEA based approach is shown in Fig. 1.

\section{B. Settings of the Proposed Approach}

The techniques used in this study were developed and implemented using FORTRAN language. On all optimization runs, the population size and the maximum number of generations were selected as 200 and 500 respectively. The maximum size of the Pareto-optimal set was set as 30 solutions. If the number of the nondominated Pareto optimal solutions exceeds this bound, the clustering technique is called. Crossover and mutation probabilities were selected as 0.9 and 0.01 respectively in all optimization runs.

\section{Results AND Discussions}

\section{A. Wale and Hale 6-bus Test System}

In this study, the proposed approach was tested on the Wale and Hale 6 bus test system shown in Fig. 2. The detailed line data, bus data, and control variable limits are given in [17]. The system has two generators, two transformers, and two capacitor banks as shown. The number of constraints in this case is 24. At first, the $P_{L}$ and $L_{\text {index }}$ objectives are optimized individually in order to explore the extreme points of the trade-off surface and evaluate the diversity characteristics of the Pareto optimal solutions obtained by the proposed approach. The best results of $P_{L}$ and $L_{\text {index }}$ when optimized individually are given in Table 1 . Convergence of $P_{L}$ and $L_{\text {index }}$ objectives are shown in Fig. 3.

The problem is handled as a multiobjective optimization problem and the proposed approach has been implemented to optimize both objectives simultaneously. The size of Pareto optimal set is selected 30 nondominated solutions in this work. Unlike the traditional techniques such as the weighted sum and the epsilon-constraint methods, the proposed approach obtains these solutions in one single run. The diversity of the Pareto optimal set over the trade-off surface is shown in Fig. 4. Out of them, two nondominated solutions that represent the best $P_{L}$ and best $L_{\text {index }}$ are given in Table 2. It is worth mentioning that the initial load flow indicates voltage 
violation at bus 3 where $V_{3}=0.855 \mathrm{pu}$. After optimization, no violations have been observed.

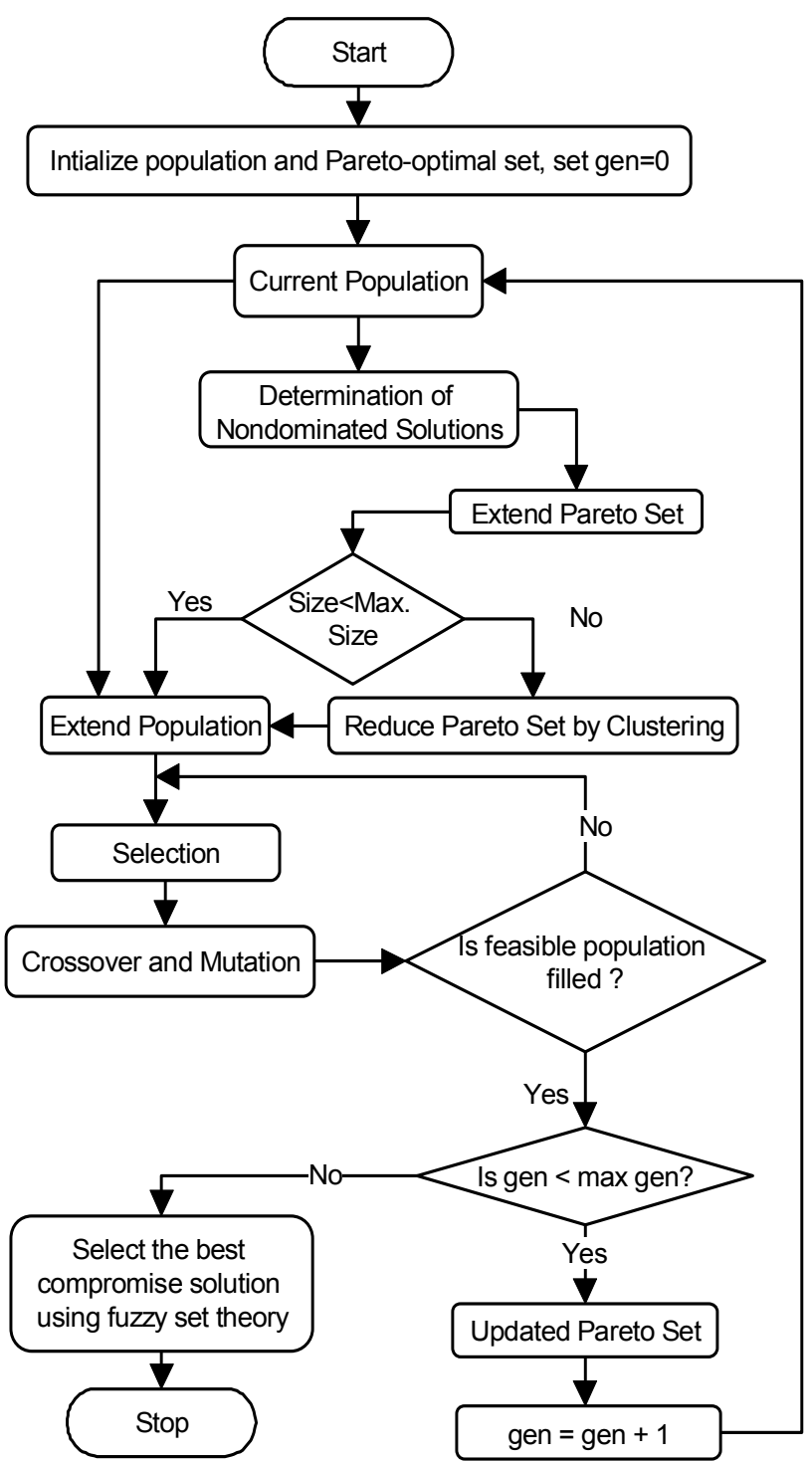

Fig. 1: Strength Pareto Evolutionary Algorithm

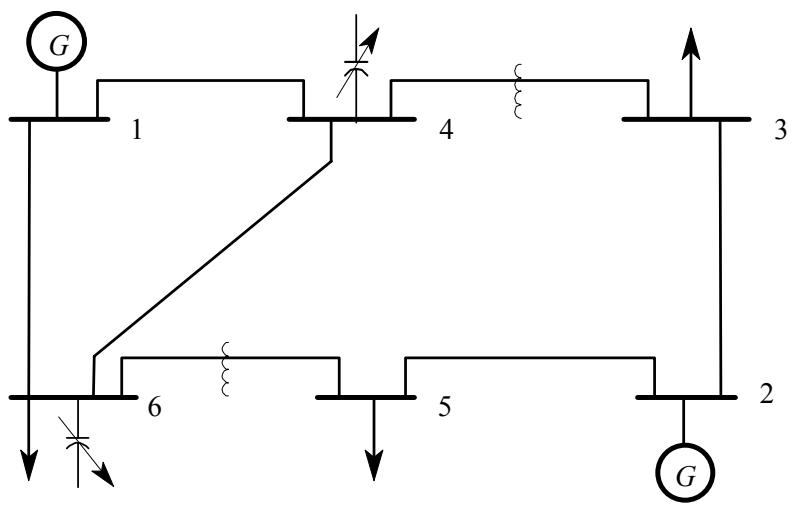

Fig. 2: Single line diagram of the 6-bus system

\begin{tabular}{cccc}
\hline$V_{G 1}$ & 1.050 & 1.098 & 1.086 \\
$V_{G 2}$ & 1.100 & 1.137 & 1.150 \\
$T_{3-4}$ & 1.025 & 0.949 & 0.973 \\
$T_{5-6}$ & 1.100 & 0.982 & 0.997 \\
$Q_{c 4}$ & 0.0 & 0.049 & 0.049 \\
$Q_{c 6}$ & 0.0 & 0.055 & 0.055 \\
\hline$P_{L}(\mathrm{MW})$ & 11.609 & $\mathbf{8 . 6 8 3}$ & 8.906 \\
$L_{\text {index }}$ & 0.2720 & 0.2348 & $\mathbf{0 . 2 3 0 4}$ \\
\hline
\end{tabular}

TABLE 2: THE RESULTS OF PROPOSED APPROACH

\begin{tabular}{cccc}
\hline & Best $P_{L}$ & Best $L_{\text {index }}$ & Best Compromise \\
\hline$V_{G 1}$ & 1.096 & 1.081 & 1.091 \\
$V_{G 2}$ & 1.139 & 1.150 & 1.150 \\
$T_{3-4}$ & 0.949 & 1.027 & 0.957 \\
$T_{5-6}$ & 0.982 & 0.985 & 0.984 \\
$Q_{c 4}$ & 0.050 & 0.050 & 0.050 \\
$Q_{c 6}$ & 0.055 & 0.055 & 0.055 \\
\hline$P_{L}(\mathrm{MW})$ & $\mathbf{8 . 6 8 8}$ & 9.192 & 8.730 \\
$L_{\text {index }}$ & 0.2344 & $\mathbf{0 . 2 3 0 6}$ & 0.2308 \\
\hline
\end{tabular}

The comparison of the average value of the run time per Pareto-optimal solution over 10 different optimization runs of the traditional weighted sum method and the proposed approach is given in Table 3. It is quiet evident that the run time of proposed approach is much less than that of the weighted sum method as it produces all the Pareto-optimal solutions in one single run.

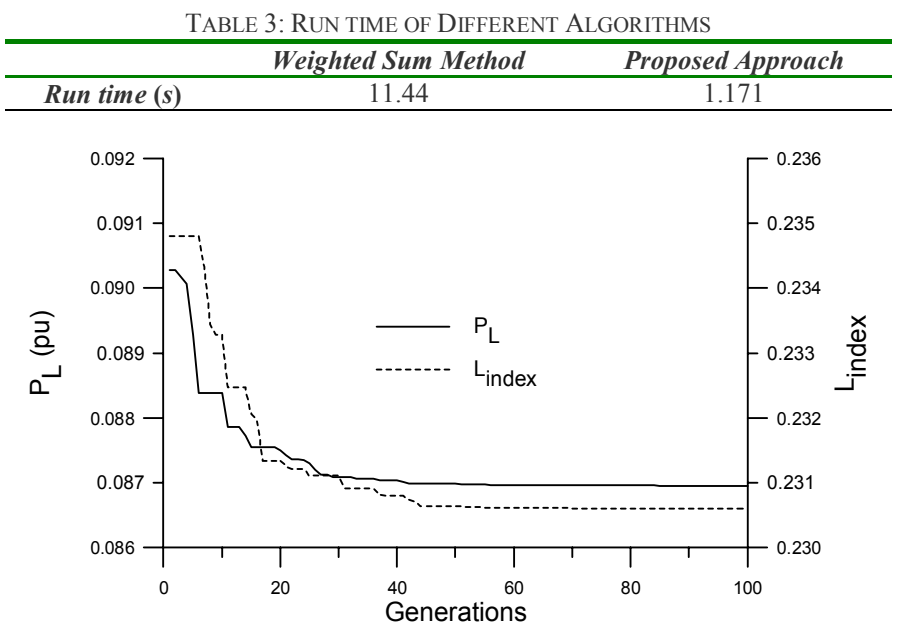

Fig. 3: Convergence of the objectives in individual optimization for the 6-bus test system

The membership functions given in (26) and (27) were used to evaluate each member of the Pareto-optimal set. Then, the best compromise solution was extracted. The best compromise solution is shown in Fig. 3 and given in Table 2.

TABLE 1: THE BEST SOLUTIONS FOR INDIVIDUAL OPTIMIZATION Initial [21] Best $P_{L} \quad$ Best $L_{\text {in }}$ 


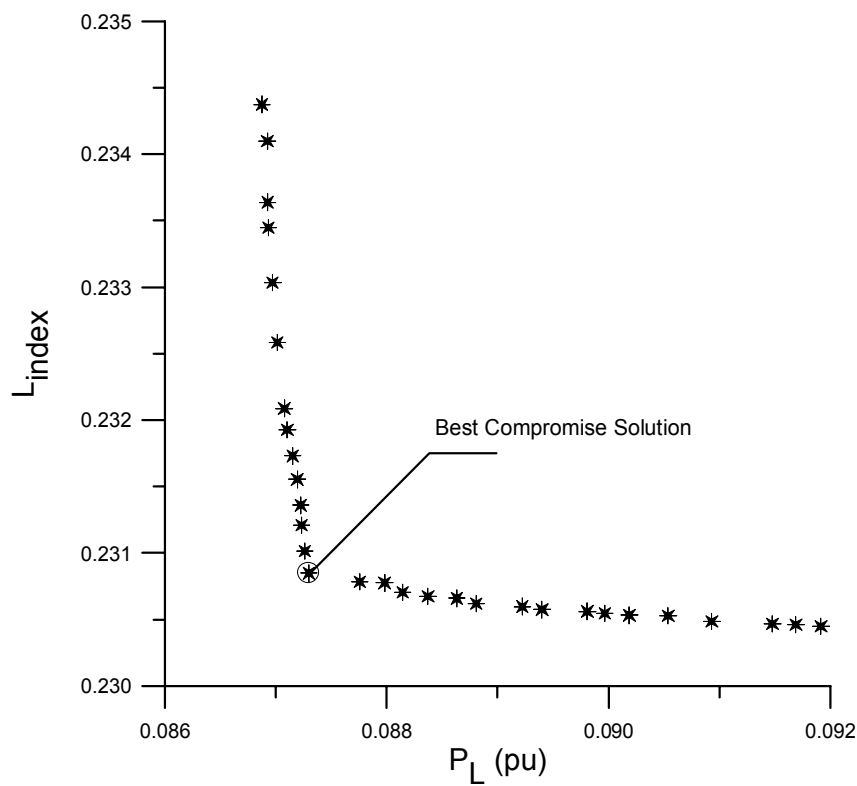

Fig. 4: Pareto-optimal front of the proposed approach for the 6-bus test system

\section{B. IEEE 30-bus 6-generator Test System}

The proposed approach was tested on the standard IEEE 30-bus 6-generator test system in order to investigate its effectiveness. The single-line diagram of the IEEE test system is shown in Fig. 5 and the detailed data are given in [18]. The system has six generators at buses $1,2,5,8,11$, and 13 and four transformers with off-nominal tap ratio in lines 6-9, 6-10, $4-12$, and 27-28. The lower voltage magnitude limits at all buses are $0.95 \mathrm{pu}$ and the upper limits are $1.1 \mathrm{pu}$ for generator buses $2,5,8,11$, and 13 , and 1.05 pu for the remaining buses including the reference bus 1 . The lower and upper limits of the transformer tappings are 0.9 and 1.1 pu respectively. The initial settings of the control variables and the initial values of objective functions are given in Table 4. The number of constraints in this case is 100 .

At first, the $P_{L}$ and $L_{\text {index }}$ objectives are optimized individually and the best results of $P_{L}$ and $L_{\text {index }}$ are given in Table 4. Convergence of both objectives is shown in Fig. 6.

The problem was handled as a multiobjective optimization problem where both objectives were optimized simultaneously with the proposed approach. The diversity of the Pareto optimal set over the trade-off surface is shown in Fig. 7. It is worth mentioning that the Pareto optimal set has 30 nondominated solutions. Out of them, two nondominated solutions that represent the best $P_{L}$ and best $L_{\text {index }}$ are given in Table 5. In addition, the best compromise solution is shown in Fig. 7 and given in Table 5.

\section{CONCLUSION}

In this paper, a new approach based on the strength Pareto evolutionary algorithm has been presented and applied to multiobjective VAR dispatch optimization problem. The problem has been formulated as multiobjective optimization problem with real power loss and voltage stability objectives. A hierarchical clustering technique is implemented to provide the operator with a representative and manageable Paretooptimal set without destroying the characteristics of the trade- off front. Moreover, a fuzzy-based mechanism is employed to extract the best compromise solution over the trade-off curve. The results show that the proposed approach is efficient for solving multiobjective VAR dispatch problem where multiple Pareto optimal solutions can be found in one simulation run. In addition, the nondominated solutions obtained are well distributed and have satisfactory diversity characteristics.

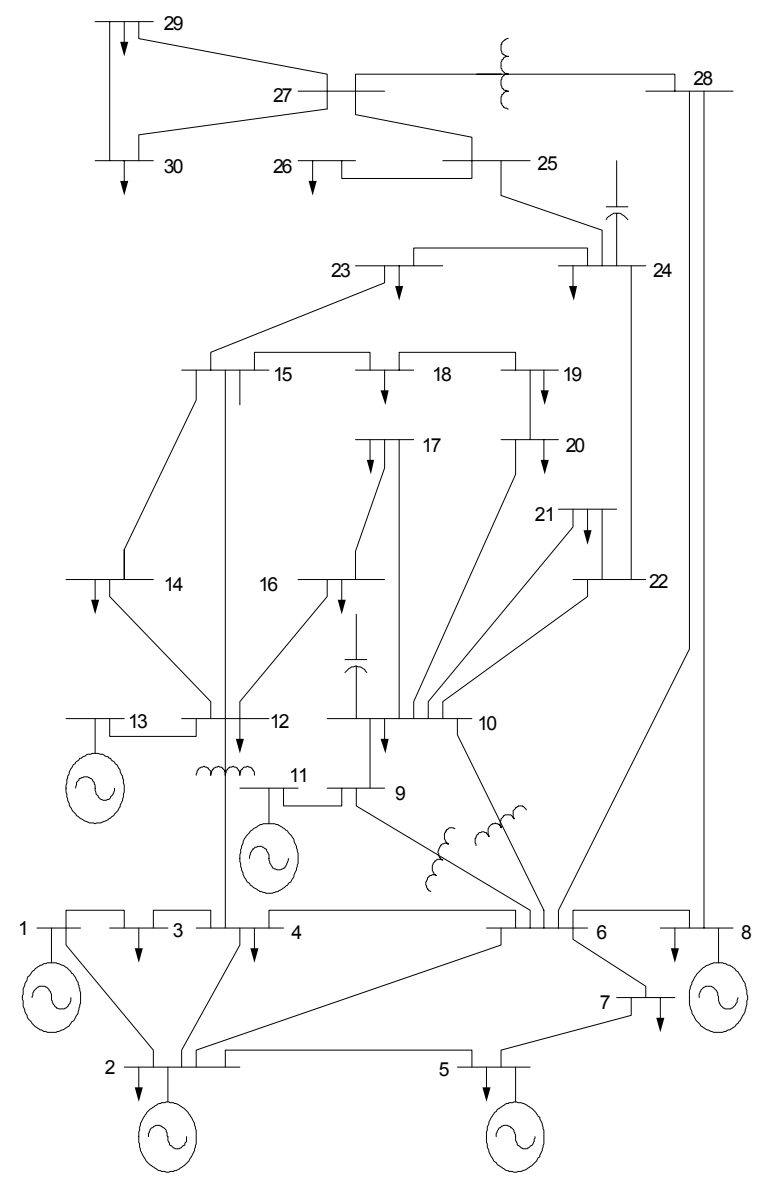

Fig. 5: Single-line diagram of IEEE 30-bus system

TABLE 4: THE BEST SOLUTIONS FOR INDIVIDUAL OPTIMIZIZATION

\begin{tabular}{cccc}
\hline & Initial $[18]$ & Best $P_{L}$ & Best $L_{\text {index }}$ \\
\hline$V_{G 1}$ & 1.050 & 1.050 & 1.044 \\
$V_{G 2}$ & 1.045 & 1.041 & 1.045 \\
$V_{G 5}$ & 1.010 & 1.018 & 1.063 \\
$V_{G 8}$ & 1.010 & 1.017 & 1.015 \\
$V_{G 11}$ & 1.050 & 1.084 & 1.031 \\
$V_{G 13}$ & 1.050 & 1.079 & 1.046 \\
$T_{6-9}$ & 0.978 & 1.002 & 0.963 \\
$T_{6-10}$ & 0.969 & 0.951 & 0.912 \\
$T_{4-12}$ & 0.932 & 0.990 & 0.929 \\
$T_{27-28}$ & 0.968 & 0.940 & 0.933 \\
\hline$P_{L}(\mathrm{MW})$ & 5.3786 & $\mathbf{5 . 1 1 7}$ & 6.002 \\
$L_{\text {index }}$ & 0.1453 & 0.1418 & $\mathbf{0 . 1 3 9 7}$ \\
\hline
\end{tabular}




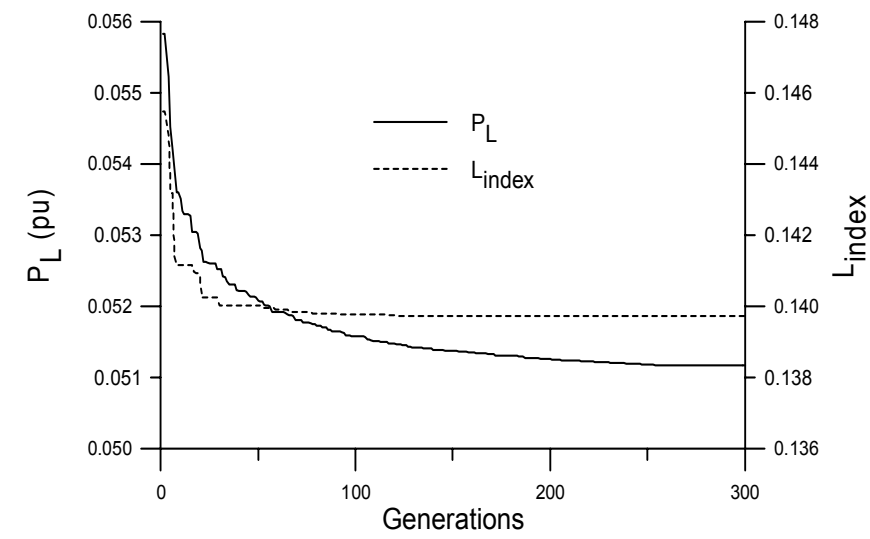

Fig. 6: Convergence of the objectives in individual optimization for the 30-bus test system

\begin{tabular}{cccc} 
& \multicolumn{3}{c}{ TABLE 5: THE RESULTS OF PROPOSED APPROACH } \\
\hline & Best $P_{L}$ & Best $L_{\text {index }}$ & Best Compromise \\
\hline$V_{G 1}$ & 1.050 & 1.050 & 1.050 \\
$V_{G 2}$ & 1.044 & 1.050 & 1.044 \\
$V_{G 5}$ & 1.025 & 1.074 & 1.026 \\
$V_{G 8}$ & 1.026 & 1.018 & 1.028 \\
$V_{G 11}$ & 1.095 & 1.025 & 1.026 \\
$V_{G 13}$ & 1.060 & 1.068 & 1.058 \\
$T_{6-9}$ & 1.048 & 0.961 & 0.961 \\
$T_{6-10}$ & 0.915 & 0.919 & 0.919 \\
$T_{4-12}$ & 0.962 & 0.956 & 0.956 \\
$T_{27-28}$ & 0.944 & 0.938 & 0.940 \\
\hline$P_{L}(\mathrm{MW})$ & $\mathbf{5 . 1 2 3}$ & 6.071 & 5.258 \\
$L_{\text {index }}$ & 0.1411 & $\mathbf{0 . 1 3 9 7}$ & 0.1399 \\
\hline
\end{tabular}

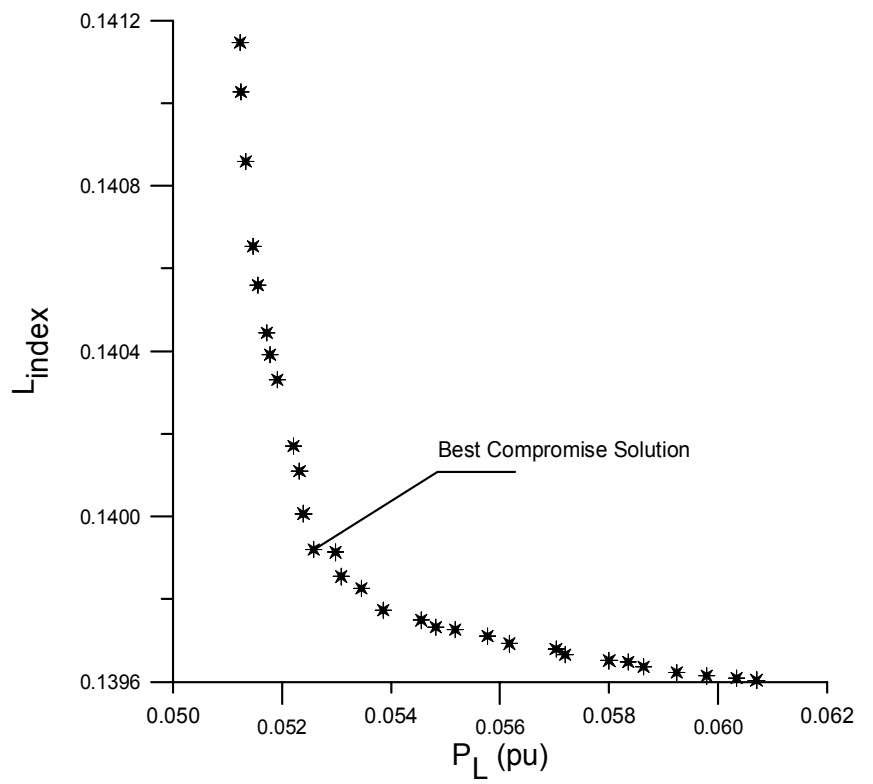

Fig. 7: Pareto-optimal front of the proposed approach for the 30-bus test system

\section{ACKNOWLEDGMENT}

The author acknowledges the support of King Fahd Univ. of Petroleum \& Minerals via funded project \# SAB/2005-07.

\section{REFERENCES}

[1] M. O. Mansour and T. M. Abdel-Rahman, "Non-Linear VAR Optimization Using Decomposition and Coordination," IEEE Trans. on Power Apparatus and Systems, PAS-103, No. 2, 1984, pp. 246-255.

[2] K. R. C. Mamandur and R. D. Chenoweth, "Optimal Control of Reactive Power Flow for Improvements in Voltage profiles and for Real Power Loss Minimization," IEEE Trans. on Power Apparatus and Systems, Vol. PAS-100, No. 7, 1981, pp. 3185-3193.

[3] K. Iba, "Reactive Power Optimization by Genetic Algorithm," IEEE Trans. on Power Systems, Vol. 9, No. 2, 1994, pp. 685-692.

[4] L. L. Lai and J. T. Ma, " Application of Evolutionary Programming to Reactive Power Planning-Comparison with Nonlinear Programming Approach," IEEE Trans. on Power Systems, Vol. 12, No. 1, 1997, pp. 198-206.

[5] Y. T. Hsiao and H. D. Chiang, "Applying Network Window Schema and a Simulated Annealing Technique to Optimal VAR Planning in LargeScale Power systems," Electric Power Systems Research, 22, 2000, pp. $1-8$.

[6] D. Gan, Z. Qu, and H. Cai, "Large-Scale VAR Optimization and Planning by Tabu Search," Electric Power Systems Research, 39, 1996, pp. 195-204.

[7] N. Grudinin, "Reactive Power Optimization Using Successive Quadratic Programming Method," IEEE Trans. on Power Systems, Vol. 13, No. 4, 1998, pp. 1219-1225.

[8] Y. T. Hsaio, H. D. Chaing, C. C. Liu, and Y. L. Chen, "A Computer Package for Optimal Multi-objective VAR Planning in Large Scale Power Systems," IEEE Trans. on Power Systems, Vol. 9, No. 2, 1994, pp. 668-676.

[9] C. M. Fonseca and P. J. Fleming, "An Overview of Evolutionary Algorithms in Multiobjective Optimization," Evolutionary Computation, Vol. 3, No. 1, 1995, pp. 1-16.

[10] C. A. C. Coello, "A Comprehensive Survey of Evolutionary-Based Multiobjective Optimization Techniques," Knowledge and Information Systems, Vol. 1, No. 3, 1999, pp. 269-308.

[11] E. Zitzler and L. Thiele, "An Evolutionary Algorithm for Multiobjective optimization: The Strength Pareto Approach,” Swiss Federal Institute of Technology, TIK-Report, No. 43, 1998.

[12] M. A. Abido and J. M. Bakhashwain, "Optimal VAR Dispatch Using a Multiobjective Evolutionary Algorithm" International Journal of Electrical Power \& Energy Systems, Vol. 27, No. 1, January 2005, pp. 13-20.

[13] C. Belhadj, R. Mohamedi, S. Lefebvre, P.-J Lagace,and Do Xuan-Dai, "Voltage Stability Modelling and Real-Time Monitoring Using Expert System for Operation Assistance," IEEE Trans. on Power Systems, Vol. 11, No. 2, May 1996, pp. 1037 - 1045.

[14] C.Belhadj, M. A. Abido, "An optimized fast voltage stability indicator", IEEE Budapest Power Tech '99 Conference, Budapest, Hungary, August 29- September 2, 1999, BPT99-363-12 J.

[15] N. Morse, "Reducing the Size of Nondominated Set: Pruning by Clustering," Computers and Operations Research, Vol. 7, No. 1-2, 1980, pp. 55-66.

[16] J. S. Dhillon, S. C. Parti, and D. P. Kothari, "Stochastic Economic Emission Load Dispatch,” Electric Power Systems Research, 26, 1993, pp. 197-186.

[17] Z. Wang, D. Peng, Q. Feng, H. Liu, and D. C. Yu, "A Non-Incremental Model for Optimal Control of Reactive Power Flow," Electric Power Systems Research, Vol. 39, 1996, pp. 153-159.

[18] O. Alsac and B. Stott, "Optimal Load Flow with Steady State Security," IEEE Trans. on Power Apparatus and Systems, Vol. PAS-93, 1974, pp. 745-751. 